\title{
HYGROTHERMO-MECHANICAL BEHAVIOUR OF LAYERED COMPOSITE PLATES
}

\author{
TRAN ICH ThINH \\ Hanoi University of Technology
}

\begin{abstract}
In this paper, a hygrothermomechanical behaviour of simply supported, composite layered rectangular plates is presented. The analysis is based on use of the Finite Element (FE) method and the full third-order displacement theory. Numerical examples are presented for symmetrically in-axis $\left(0^{\circ} / 90^{\circ} / 90^{\circ} / 0^{\circ}\right)$ and off-axis $\left(45^{\circ} /-45^{\circ} /\right.$ $-45^{\circ} / 45^{\circ}$ ) layered rectangular plates.
\end{abstract}

\section{Introduction}

Many composite material plates not only involve anisotropy, multilayered considerations and transverse shear deformation, but also have hygrothermal effects; which can be very important. Thus, for the final design of these structures, transverse shear deformation and hygrothermal effects must be included.

Relatively few studies have been done regarding hygrothermal effects in thin and thick composite plates [1], [2], [3].

If transverse shear deformation effects are important, and if there is a hygrothermal environment present, then obtaining a solution through solving the partial differential equations with the attendant nonhomogeneous boundary conditions is a major challenge. Even if the solution is obtained it requires a major effort. Therefore, Finite Element Method is more convenient for use in design and analysis of composite plate structures.

\section{Laminate deformation and hygrothermal stress}

The present analysis is based upon a displacement field in which the displacement components $u, v, w$ are all of third-order in the thickness coordinate $z$ [5], [6]:

$$
(u, v, w)=\left(u^{0}, v^{0}, w^{0}\right)+z\left(\psi_{x}, \psi_{y}, \psi_{z}\right)+z^{2}\left(\xi_{x}, \xi_{y}, \xi_{z}\right)+z^{3}\left(\phi_{x}, \phi_{y}, \phi_{z}\right) .
$$

where superscript " 0 " denotes mid-plane displacements.

The strain components are related to the mid-plane displacements of the laminated plate as:

$$
\begin{aligned}
\left(\varepsilon_{x}, \varepsilon_{y}, \varepsilon_{z}\right) & =\left(\varepsilon_{x}^{0}, \varepsilon_{y}^{0}, \varepsilon_{z}^{0}\right)+z\left(\kappa_{x}, \kappa_{y}, \kappa_{z}\right)+z^{2}\left(\chi_{x}, \chi_{y}, \chi_{z}\right)+z^{3}\left(\eta_{x}, \eta_{y}, \eta_{z}\right) \\
\left(\gamma_{y z}, \gamma_{x z}, \gamma_{x y}\right) & =\left(\gamma_{y z}^{0}, \gamma_{x z}^{0}, \gamma_{x y}^{0}\right)+z\left(\kappa_{y z}, \kappa_{x z}, \kappa_{x y}\right)+z^{2}\left(\chi_{y z}, \chi_{x z}, \chi_{x y}\right)+z^{3}\left(\eta_{y z}, \eta_{x z}, \eta_{x y}\right)
\end{aligned}
$$


where:

$$
\begin{aligned}
\varepsilon_{x}^{0} & =u_{, x}^{0} ; \quad \varepsilon_{y}^{0}=v_{, y}^{0} ; \quad \varepsilon_{z}^{0}=\psi_{z} \\
\gamma_{y z}^{0} & =w_{, y}^{0}+\psi_{y} ; \quad \gamma_{x z}^{0}=w_{, z}^{0}+\psi_{x} ; \quad \gamma_{x y}^{0}=u_{, y}^{0}+v_{, x}^{0} ; \\
\kappa_{x} & =\psi_{x, x} ; \quad \kappa_{y}=\psi_{y, y} ; \quad \kappa_{z}=2 \xi_{z} ; \\
\kappa_{y z} & =\psi_{z, y}+2 \xi_{y} ; \quad \kappa_{x z}=\psi_{z, x}+2 \xi_{x} ; \quad \kappa_{x y}=\psi_{x, y}+\psi_{y, x} ; \\
\chi_{x} & =\xi_{x^{\prime} x} ; \quad \chi_{y}=\xi_{y^{\prime} y} ; \quad \chi_{z}=3 \phi_{x} ; \\
\chi_{y z} & =\xi_{z^{\prime} y}+3 \phi_{y} ; \quad \chi_{x z}=\xi_{z^{\prime} x}+3 \phi_{x} ; \quad \chi_{x y}=\xi_{x^{\prime} y}+\xi_{y^{\prime} x} \\
\eta_{x} & =\phi_{x, x} ; \quad \eta_{y}=\phi_{y, y} ; \quad \eta_{z}=0 \\
\eta_{y z} & =\phi_{z^{\prime} y} ; \quad \eta_{x z}=\phi_{z^{\prime} x} ; \quad \eta_{x y}=\phi_{x, y}+\phi_{y, x} .
\end{aligned}
$$

The stress-strain relation, including hygrothermal effects, is given in structural coordinates $(x-y-z)$ by [4], [5]:

$$
\left\{\begin{array}{l}
\sigma_{x x} \\
\sigma_{y y} \\
\sigma_{z z} \\
\sigma_{y z} \\
\sigma_{x z} \\
\sigma_{x y}
\end{array}\right\}_{k}=\left[\begin{array}{cccccc}
C_{11}^{\prime} & C_{12}^{\prime} & C_{13}^{\prime} & 0 & 0 & C_{16}^{\prime} \\
C_{12}^{\prime} & C_{22}^{\prime} & C_{23}^{\prime} & 0 & 0 & C_{26}^{\prime} \\
C_{13}^{\prime} & C_{23}^{\prime} & C_{33}^{\prime} & 0 & 0 & C_{36}^{\prime} \\
0 & 0 & 0 & C_{44}^{\prime} & C_{45}^{\prime} & 0 \\
0 & 0 & 0 & C_{54}^{\prime} & C_{55}^{\prime} & 0 \\
C_{16}^{\prime} & C_{26}^{\prime} & C_{36}^{\prime} & 0 & 0 & C_{66}^{\prime}
\end{array}\right]_{k}\left\{\begin{array}{l}
\varepsilon_{x x}-\varepsilon_{x x}^{T}-\varepsilon_{x x}^{M} \\
\varepsilon_{y y}-\varepsilon_{y y}^{T}-\varepsilon_{y y}^{M} \\
\varepsilon_{z z}-\varepsilon_{z z}^{T}-\varepsilon_{z z}^{M} \\
\gamma_{y z} \\
\gamma_{x z} \\
\gamma_{x y}-\gamma_{x y}^{T}-\gamma_{x y}^{M}
\end{array}\right\}_{k}
$$

where $C_{i j}^{\prime}$ are the stiffness coefficients of material layers in reference axes $(x, y, z)$; $\varepsilon_{x x}^{T}, \varepsilon_{y y}^{T}, \varepsilon_{z z}^{T}, \gamma_{x y}^{T}$ and $\varepsilon_{x x}^{M}, \varepsilon_{y y}^{M}, \varepsilon_{z z}^{M}, \gamma_{x y}^{M}$ are the thermal and the hygroscopic strains respectively.

The relation between $C_{i j}^{\prime}$ and $C_{i j}$ is expressed by:

$$
\left[\mathbf{C}^{\prime}\right]_{k}=\left[T_{\sigma}^{-1}\right]_{k}[\mathbf{C}]_{k}\left[T_{\varepsilon}\right]_{k}
$$

where: $C_{i j}$ are the elastic coefficients of layer material in principal axes - $(1,2,3)$. Clearly the $C_{i j}$ can be written in terms of the engineering constants. $T_{\sigma}^{-1}$ is inverse matrix of stress transformation matrix $T_{\sigma} ; T_{\varepsilon}$ is strain transformation matrix.

The free hygroscopic strains in each ply are defined as:

$$
\begin{aligned}
\left(\varepsilon_{x x}^{M}, \varepsilon_{y y}^{M}, \varepsilon_{z z}^{M}, 0,0, \gamma_{x y}^{M}\right)_{k}^{t} & =T_{\varepsilon}\left(\varepsilon_{11}^{M}, \varepsilon_{22}^{M}, \varepsilon_{33}^{M}, 0,0,0\right)_{k}^{t} \\
& =T_{\varepsilon}\left(\beta_{11}, \beta_{22}, \beta_{33}, 0,0,0\right)_{k}^{t} m(z, t)
\end{aligned}
$$

where: " $t$ " signifies matrix transpose; $\beta_{i i}(i=1,2,3)$ are the coefficients of hygroscopic expansion; $m$ is the change in moisture concentration refering to a "moisturefree" environment. The thermal strains in each ply are defined by replacing $M$ by $T, \beta_{i i}$ by $\alpha_{i i}$ and $m(z, t)$ by $\Delta T$ in the (2.6). 
By integrating the stresses over the plate thickness, the generalized resultantstress strain relations can then be expressed in the following matrix form [6]:

$$
\begin{aligned}
\left\{\begin{array}{c}
\{N\} \\
\{M\} \\
\{S\} \\
\{P\}
\end{array}\right\}= & {\left[\begin{array}{llll}
\mathbf{A} & \mathbf{B} & \mathbf{D} & \mathbf{E} \\
\mathbf{B} & \mathbf{D} & \mathbf{E} & \mathbf{F} \\
\mathbf{D} & \mathbf{E} & \mathbf{F} & \mathbf{G} \\
\mathbf{E} & \mathbf{F} & \mathbf{G} & \mathbf{H}
\end{array}\right]\left\{\begin{array}{l}
\left\{\varepsilon^{0}\right\} \\
\{k\} \\
\{\chi\} \\
\{\eta\}
\end{array}\right\}-\left\{\begin{array}{l}
\left\{N^{T}\right\} \\
\left\{M^{T}\right\} \\
\left\{S^{T}\right\} \\
\left\{P^{T}\right\}
\end{array}\right\}-\left\{\begin{array}{l}
\left\{N^{M}\right\} \\
\left\{M^{M}\right\} \\
\left\{S^{M}\right\} \\
\left\{P^{M}\right\}
\end{array}\right\} } \\
& \left\{\begin{array}{l}
\{Q\} \\
\{V\} \\
\{R\} \\
\{W\}
\end{array}\right\}=\left[\begin{array}{llll}
\mathbf{A}^{\prime} & \mathbf{B}^{\prime} & \mathbf{D}^{\prime} & \mathbf{E}^{\prime} \\
\mathbf{B}^{\prime} & \mathbf{D}^{\prime} & \mathbf{E}^{\prime} & \mathbf{F}^{\prime} \\
\mathbf{D}^{\prime} & \mathbf{E}^{\prime} & \mathbf{F}^{\prime} & \mathbf{G}^{\prime} \\
\mathbf{E}^{\prime} & \mathbf{F}^{\prime} & \mathbf{G}^{\prime} & \mathbf{H}^{\prime}
\end{array}\right]\left\{\begin{array}{c}
\left\{\gamma^{0}\right\} \\
\left\{k^{\prime}\right\} \\
\left\{\chi^{\prime}\right\} \\
\left\{\eta^{\prime}\right\}
\end{array}\right\}
\end{aligned}
$$

in which

$$
\begin{gathered}
\left(A_{i j}, B_{i j}, D_{i j}, E_{i j}, F_{i j}, G_{i j}, H_{i j}\right)=\sum_{k=1}^{n} \int_{h_{k-1}}^{h_{k}}\left(C_{i j}^{\prime}\right)_{k}\left(1, z, z^{2}, z^{3}, z^{4}, z^{5}, z^{6}\right) d z ; \\
(i, j=1,2,3,6) \\
\left(A_{i j}^{\prime}, B_{i j}^{\prime}, D_{i j}^{\prime}, E_{i j}^{\prime}, F_{i j}^{\prime}, G_{i j}^{\prime}, H_{i j}^{\prime}\right)=\sum_{k=1}^{n} \int_{h_{k-1}}^{h_{k}}\left(C_{i j}^{\prime}\right)_{k}\left(1, z, z^{2}, z^{3}, z^{4}, z^{5}, z^{6}\right) d z ; \\
(i, j=4,5)
\end{gathered}
$$

and $\left\{\varepsilon^{0}\right\}=\left(\varepsilon_{x}^{0} \varepsilon_{y}^{0} \varepsilon_{z}^{0} \gamma_{x y}^{0}\right)^{t} ;\{\kappa\}=\left(\kappa_{x} \kappa_{y} \kappa_{z} \kappa_{x y}\right)^{t} ;\{\chi\}=\left(\chi_{x} \chi_{y} \chi_{z} \chi_{x y}\right)^{t}$;

$\{\eta\}=\left(\begin{array}{llll}\eta_{x} & \eta_{y} & 0 & \eta_{x y}\end{array}\right)^{t} ;\left\{\gamma^{0}\right\}=\left(\begin{array}{ll}\gamma_{y z}^{0} & \gamma_{x z}^{0}\end{array}\right)^{t} ;\left\{\kappa^{\prime}\right\}=\left(\kappa_{y z} \kappa_{x z}\right)^{t} ;\left\{\chi^{\prime}\right\}=\left(\chi_{y z} \chi_{x z}\right)^{t} ;$ $\left\{\eta^{\prime}\right\}=\left(\eta_{y z} \eta_{x z}\right)^{t}$

The hygroscopic generalized resultants are determined by:

$$
\begin{aligned}
\left\{\left\{N^{M}\right\},\left\{M^{M}\right\},\left\{S^{M}\right\},\left\{P^{M}\right\}\right\} & =\left\{\begin{array}{llll}
N_{x}^{N} & M_{x}^{M} & S_{x}^{M} & P_{x}^{M} \\
N_{y}^{M} & M_{y}^{M} & S_{y}^{M} & P_{y}^{M} \\
N_{z}^{M} & M_{z}^{M} & S_{z}^{M} & P_{z}^{M} \\
N_{x y}^{M} & M_{x y}^{M} & S_{x y}^{M} & P_{x y}^{M}
\end{array}\right\} \\
& =\int_{-h / 2}^{h / 2} \sum_{k=1}^{n}\left[C_{i j}^{\prime}\right]_{k}\left[\begin{array}{c}
\beta_{x x} \\
\beta_{y y} \\
\beta_{z z} \\
\beta_{x y}
\end{array}\right]_{k} m(z, t)\left(1, z, z^{2}, z^{3}\right) d z
\end{aligned}
$$

The thermal generalized resultants are determined by replacing $M$ by $T ; \beta_{i j}$ by $\alpha_{i j}(i j=x x, y y, z z$ and $x y)$ and $m(z, t)$ by $\Delta T$ in the (2.10). 


\section{Finite element formulation}

The FE method was chosen to solve the bending problem of rectangular layered plates under lateral and hygrothermal loads. Isoparametric Lagrangian elements with 9 nodes are used. The generalized displacement vector can be written as

$$
\mathbf{a}^{t}=\left\{\mathbf{d}_{1}^{t} \mathbf{d}_{2}{ }^{t} \ldots \mathbf{d}_{N e}^{t}\right\}
$$

where $\mathrm{d}$ stands for $u_{o}, v_{o}, w_{o}, \psi_{x}, \psi_{y}, \psi_{z}, \xi_{x}, \xi_{y}, \xi_{z}, \phi_{x}, \phi_{y}$ or $\phi_{z} ; N_{e}$ is the number of elements.

Thus, with the aid of (3.1), it is possible to write the expression for the total potential energy for an element " $e$ ":

$$
\begin{aligned}
& \Pi_{e}=\frac{1}{2} \iint_{S_{e}}\left\{\mathbf{a}^{t} \mathbf{B}_{1}^{t} \mathbf{A B}_{1} \mathbf{a}+\mathbf{a}^{t} \mathbf{B}_{1}^{t} \mathbf{B B}_{2} \mathbf{a}+\mathbf{a}^{t} \mathbf{B}_{1}^{t} \mathbf{D B}_{3} \mathbf{a}+\mathbf{a}^{t} \mathbf{B}_{1}^{t} \mathbf{E B}_{4} \mathbf{a}\right. \\
& +\mathbf{a}^{t} \mathbf{B}_{2}^{t} \mathbf{B B}_{1} \mathbf{a}+\mathbf{a}^{t} \mathbf{B}_{2}^{t} \mathbf{D B}_{2} a+\mathbf{a}^{t} \mathbf{B}_{2}^{t} \mathbf{E B}_{3} a+\mathbf{a}^{t} \mathbf{B}_{2}^{t} \mathbf{F B}_{4} \mathbf{a}+\mathbf{a}^{t} \mathbf{B}_{3}^{t} \mathbf{D B}_{1} \mathbf{a} \\
& +\mathbf{a}^{t} \mathbf{B}_{3}^{t} \mathbf{E B}_{2} \mathbf{a}+\mathbf{a}^{t} \mathbf{B}_{3}^{t} \mathbf{F B}_{3} \mathbf{a}+\mathbf{a}^{t} \mathbf{B}_{3}^{t} \mathbf{G B}_{4} \mathbf{a}+\mathbf{a}^{t} \mathbf{B}_{4}^{t} \mathbf{E B}_{1} \mathbf{a}+\mathbf{a}^{t} \mathbf{B}_{4}^{t} \mathbf{F B}_{2} \mathbf{a} \\
& +\mathbf{a}^{t} \mathbf{B}_{4}^{t} \mathbf{G B}_{3} \mathbf{a}+\mathbf{a}^{t} \mathbf{B}_{4}^{t} \mathbf{H B}_{4} \mathbf{a}+\mathbf{a}^{t} \mathbf{B}_{1}^{\prime t} \mathbf{A}^{\prime} \mathbf{B}^{\prime}{ }_{1} \mathbf{a}+\mathbf{a}^{t} \mathbf{B}^{\prime t} \mathbf{B}^{\prime} \mathbf{B}^{\prime}{ }_{2} \mathbf{a}+\mathbf{a}^{t} \mathbf{B}_{1}^{\prime t} \mathbf{D}^{\prime} \mathbf{B}^{\prime} \mathbf{a}
\end{aligned}
$$

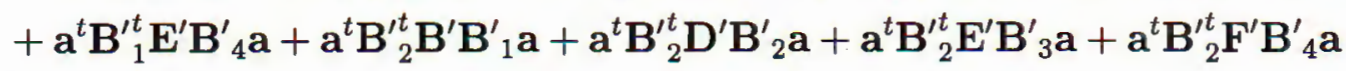

$$
\begin{aligned}
& +\mathbf{a}^{t} \mathbf{B}_{3}^{\prime t} \mathbf{D}^{\prime} \mathbf{B}_{1}^{\prime} \mathbf{a}+\mathbf{a}^{t} \mathbf{B}^{\prime t}{ }_{3} \mathbf{E}^{\prime} \mathbf{B}_{2}^{\prime} \mathbf{a}+\mathbf{a}^{t} \mathbf{B}_{3}^{\prime t} \mathbf{F}^{\prime} \mathbf{B}_{3}^{\prime} \mathbf{a}+\mathbf{a}^{t} \mathbf{B}^{\prime t}{ }_{3} \mathbf{G}^{\prime} \mathbf{B}^{\prime}{ }_{4} \mathbf{a} \\
& \left.+\mathbf{a}^{t} \mathbf{B}_{4}^{\prime t} \mathbf{E}^{\prime} \mathbf{B}^{\prime}{ }_{1} \mathbf{a}+\mathbf{a}^{t} \mathbf{B}_{4}^{\prime t} \mathbf{F}^{\prime} \mathbf{B}_{2}^{\prime} \mathbf{a}+\mathbf{a}^{t} \mathbf{B}_{4}^{\prime t} \mathbf{G}^{\prime} \mathbf{B}_{3}^{\prime} \mathbf{a}+\mathbf{a}^{t} \mathbf{B}^{\prime}{ }_{4} \mathbf{H}^{\prime} \mathbf{B}^{\prime}{ }_{4} \mathbf{a}\right\} d S \\
& -\iint_{S_{e}}\left\{\mathbf{a}^{t} \mathbf{B}_{1}^{t}\left(N^{T}+N^{M}\right)+\mathbf{a}^{t} \mathbf{B}_{2}^{t}\left(M^{T}+M^{M}\right)\right. \\
& \left.+\mathbf{a}^{t} \mathbf{B}_{3}^{t}\left(S^{T}+S^{M}\right)+\mathbf{a}^{t} \mathbf{B}_{4}^{t}\left(P^{T}+P^{M}\right)\right\} d S \\
& +\iint_{S_{e}}\left\{T^{*}+M^{*}+\overline{M T}^{*}-p(x, y) w(x, y)\right\} d S,
\end{aligned}
$$

where:

$$
\begin{aligned}
T^{*} & =\frac{1}{2} \sum_{k=1}^{n} \int_{k_{k}-1}^{h_{k}}\left[C_{i j}\right]_{k}[\alpha]_{k}^{t}[\alpha]_{k} \Delta T^{2}(z, t) d z, \\
M^{*} & =\frac{1}{2} \sum_{k=1}^{n} \int_{h_{k}-1}^{h_{k}}\left[C_{i j}\right]_{k}[\beta]_{k}^{t}[\beta]_{k} m^{2}(z, t) d z, \\
\overline{M T}^{*} & =\sum_{k=1}^{n} \int_{h_{k}-1}^{h_{k}}\left[C_{i j}\right]_{k}[\alpha]_{k}^{t}[\beta]_{k} \Delta T(z, t) m(z, t) d z .
\end{aligned}
$$


Taking the first variation of the functional

$$
\frac{\partial \Pi_{e}}{\partial \mathbf{a}}=0
$$

and assembling all elements yields the equilibrium equations system as follows:

$$
[\mathbf{K}]\{\mathbf{Q}\}=\left\{\mathbf{F}^{L}\right\}+\left\{\mathbf{F}^{M}\right\}+\left\{\mathbf{F}^{T}\right\}
$$

where $[\mathbf{K}]$ is the global stiffness matrix without displacement constraints, $\{\mathbf{Q}\}$ is the assembled nodal displacement vector; $\left\{\mathbf{F}^{L}\right\},\left\{\mathbf{F}^{M}\right\}$ and $\left\{\mathbf{F}^{T}\right\}$ are the assembled nodal force vector, generalized hygroscopic loading vector and generalized thermal loading vector, respectively.

In the present work, the $3 \times 3$ Gausian rule is used to evaluate the stiffness matrix $[\mathbf{K}]$ and generalized loading vectors $\left\{\mathbf{F}^{i}\right\},(i=L, M, T)$.

\section{Numerical results}

A simply-supported $\left(45^{\circ} /-45^{\circ} /-45^{\circ} / 45^{\circ}\right)$ rectangular plate, $(a \times b ; b / a=$ $2 ; a / h=10)$, under uniform transverse load, $p$, temperature and moisture is considered. A uniform $4 \times 4$ mesh of nine-node quadrilateral isoparametric elements (twelve-degrees-of-freedom per node) was used to model a full symmetric plate.

To efficiently effect the calculations, a computer program was developed. The output gives deflections, rotations, stress resultants, strains and stresses in the plates.

The material properties used for each lamina of the graphite-epoxy composite are as follows: $E_{1}=280 \mathrm{GPa} ; E_{2}=E_{3}=7 \mathrm{GPa} ; G_{12}=G_{13}=4.2 \mathrm{GPa} ; G_{23}=3.5 \mathrm{GPa}$; $\nu_{12}=\nu_{23}=\nu_{13}=0.25 ; a=400 \mathrm{~mm} ; p=1 \mathrm{~N} / \mathrm{mm}^{2} ; \alpha_{1}=-0.018 E-06 /{ }^{\circ} \mathrm{C}, \alpha_{2}=$ $24.3 E-06 /{ }^{\circ} \mathrm{C}, \alpha_{3}=24.3 E-06 /{ }^{\circ} \mathrm{C} ; \beta_{1}=146 E-06 / \% \mathrm{~m}, \beta_{2}=4700 E-06 / \% \mathrm{~m}$, $\beta_{3}=4700 E-06 / \% \mathrm{~m} ; \Delta m_{s}=1 \%$ and $D T=D_{z} \times t=1.29 \mathrm{~mm}^{2} ; \Delta T=50^{\circ} \mathrm{C}$. The change in moisture concentration $m(z, t)$ is calculated using the Fickian solution [5]. The temperature distribution in the $z$-direction is assumed to be constant. We studied the bending behaviour of the plate for four cases following:

- Case 1: under uniform lateral pressure: $p_{0}=1 \mathrm{MPa}$; denote by $P$

- Case 2: under uniform lateral pressure and hygroscopic loading; denote by $P+M$

- Case 3: under uniform lateral pressure and thermal loading; denote by $P+T$

- Case 4: under uniform lateral pressure and hygrothermal loading; denote by $P+M+T$

The stresses at point $(a / 4 ; b / 4)$ are presented in Table 1 . 
Tab. 1. The stresses in the $\left(45^{\circ} /-45^{\circ} /-45^{\circ} / 45^{\circ}\right)$ plate under lateral and hygrothermal loads

\begin{tabular}{|c|c|c|c|c|c|c|c|}
\hline Loading & $z / h$ & $\begin{array}{l}\sigma_{1}(\mathrm{MPa}) \\
(a / 4, b / 4)\end{array}$ & $\begin{array}{l}\sigma_{2}(\mathrm{MPa}) \\
(a / 4, b / 4)\end{array}$ & $\begin{array}{l}\sigma_{3}(\mathrm{MPa}) \\
(a / 4, b / 4)\end{array}$ & $\begin{array}{l}\sigma_{23}(\mathrm{MPa}) \\
(a / 4, b / 4)\end{array}$ & $\begin{array}{l}\sigma_{13}(\mathrm{MPa}) \\
(a / 4, b / 4)\end{array}$ & $\begin{array}{l}\sigma_{12}(\mathrm{MPa}) \\
(a / 4, b / 4)\end{array}$ \\
\hline \multirow{7}{*}{$\mathrm{P}$} & -0.5 & 36.9074 & 5.6145 & -0.1029 & 0.0279 & -1.5888 & -1.2366 \\
\hline & -0.25 & 15.2522 & 2.4893 & -0.3608 & 0.0295 & 486 & -0.1410 \\
\hline & -0.25 & 60.7515 & 1.1384 & -0.2425 & 1.8194 & -0.0736 & 0.1410 \\
\hline & 0 & 5.9098 & -0.2034 & -0.4492 & 2.6466 & -0.1040 & -0.7318 \\
\hline & 0.25 & -47.0257 & -1.5378 & -0.6969 & 1.8454 & -0.1626 & -1.5828 \\
\hline & 0.25 & -22.9770 & -2.2518 & -0.6344 & 0.0651 & 4.6136 & 1.5828 \\
\hline & 0.5 & -44.7232 & -5.1822 & -1.0306 & 0.1004 & -1.4618 & 2.6133 \\
\hline \multirow{7}{*}{$\mathrm{P}+\mathrm{M}$} & -0.5 & 42.9193 & 4.0279 & -7.5950 & 0.1004 & -1.4618 & 2.6133 \\
\hline & -0.25 & 65.0006 & 3.1405 & 6.0552 & 0.0651 & & 1.5828 \\
\hline & -0.25 & 26.8800 & 4.2723 & 5.9561 & 1.8454 & -0.1626 & -1.5828 \\
\hline & 0 & 8.2592 & 2.9810 & 10.7002 & 6466 & -0.1040 & -0.7318 \\
\hline & 0.25 & -51.0074 & 0.5125 & 6.3452 & 194 & 736 & 1410 \\
\hline & 0.25 & -12.5770 & -0.6285 & 4452 & 0295 & 186 & .1410 \\
\hline & 0.5 & -39.5956 & -7.2099 & -7.0120 & 0.0279 & -1.5888 & -1.2366 \\
\hline \multirow{7}{*}{$\mathrm{P}+\mathrm{T}$} & -0.5 & 42.9943 & 0.3099 & -23.6533 & 0.1004 & 618 & 2.6133 \\
\hline & -0.25 & 36.5133 & 8.0 & 887 & 651 & 36 & 28 \\
\hline & -0.25 & 74.4070 & 6.8908 & 19.8373 & 1.8454 & -0.1626 & -1.5828 \\
\hline & 0 & 19.9943 & 9.2439 & 34.4269 & 2.6466 & -0.1040 & -0.7318 \\
\hline & 0.25 & -41.601 & 4.2629 & 20.1272 & 1.8194 & -0.0736 & 0.1410 \\
\hline & 0.25 & -2.9436 & 3.1151 & 20.2278 & 0.0295 & 4.5486 & -0.1410 \\
\hline & 0.5 & -39.520 & -10.928 & -23.0704 & 0.0279 & -1.5888 & -1.2366 \\
\hline \multirow{7}{*}{$\mathrm{P}+\mathrm{M}+\mathrm{T}$} & -0.5 & 44.6562 & -1.2811 & -30.9568 & 104 & 18 & 33 \\
\hline & -0.25 & 43.6648 & 9.8 & 25.9394 & 0.0651 & 4.6136 & 1.5828 \\
\hline & -0.25 & 81.4037 & 8.7173 & 26.0375 & 1.8454 & -0.1626 & -1.5828 \\
\hline & 0 & 28.2535 & 12.225 & 45.1271 & 2.6466 & -0.1040 & -0.7318 \\
\hline & 0.25 & -34.604 & 6.0894 & 26.3275 & 1.8194 & -0.0736 & 0.1410 \\
\hline & 0.25 & 4.20790 & 4.9370 & 26.4284 & 0.0295 & 4.54860 & -0.1410 \\
\hline & 0.5 & -37.858 & -12.518 & -30.374 & 0.0279 & -1.5888 & -1.2366 \\
\hline
\end{tabular}

It is seen that the hygrothermal environment causes increasing in $\sigma_{1}, \sigma_{2}$ and $\sigma_{3}$ in all layers of the plate, but that shear stresses have no appreciable influence.

\section{Tensor polynomial failure criterion}

The availability of all six components of stress provides a unique opportunity 
to study the application of a three dimensional failure criterion. According to the Tsai-Wu criterion [7], ply failure occurs if the failure index (FI) exceeds 1:

$$
F I=F_{i} \sigma_{i}+F_{i j} \sigma_{i} \sigma_{j}<1 ; \quad i, j=1, \ldots, 6
$$

where $F_{i}$ and $F_{i j}$ are the strength tensors whose components can be expressed in terms of the material principal strengths. When (4.1) is expanded in principal material coordinates for an orthotropic composite material, it has the form

$$
\begin{aligned}
F I= & F_{1} \sigma_{1}+F_{2} \sigma_{2}+F_{3} \sigma_{3}+F_{11} \sigma_{1}^{2}+F_{22} \sigma_{2}^{2}+F_{33} \sigma_{3}^{2}+F_{44} \sigma_{23}^{2}+F_{55} \sigma_{13}^{2} \\
& +F_{66} \sigma_{12}^{2}+2 F_{12} \sigma_{1} \sigma_{2}+2 F_{13} \sigma_{1} \sigma_{3}+2 F_{23} \sigma_{2} \sigma_{3}<1 .
\end{aligned}
$$

in which:

$$
\begin{aligned}
& F_{11}=\frac{1}{X_{t} X_{c}} ; \quad F_{22}=\frac{1}{Y_{t} Y_{c}} ; \quad F_{33}=\frac{1}{Z_{t} Z_{c}} ; \\
& F_{44}=\frac{1}{R^{2}} ; \quad F_{55}=\frac{1}{S^{2}} ; \quad F_{66}=\frac{1}{T^{2}} ; \\
& F_{12}=\frac{-1}{2 \sqrt{X_{t} X_{c} Y_{t} Y_{c}}} ; \quad F_{13}=\frac{-1}{2 \sqrt{X_{t} X_{c} Z_{t} Z_{c}}} ; \quad F_{23}=\frac{-1}{2 \sqrt{Y_{t} Y_{c} Z_{t} Z_{c}}} ; \\
& F_{1}=\frac{1}{X_{t}}-\frac{1}{X_{c}} ; \quad F_{2}=\frac{1}{Y_{t}}-\frac{1}{Y_{c}} ; \quad F_{3}=\frac{1}{Z_{t}}-\frac{1}{Z_{c}},
\end{aligned}
$$

where $X_{t}, Y_{t}, Z_{t}$ are the lamina normal strengths in tension; $X_{c}, Y_{c}, Z_{c}$ are the lamina normal strengths in compresion and $R, S, T$ are the shear strengths in $(23,13,12)$ planes, respectively. Applying the Tsai-wu criterion for the rectangular $\left(0^{\circ} / 90^{\circ} / 90^{\circ} / 0^{\circ}\right)$ plate subjected to uniform loading and hygrothermal effects, we calculated the limit loads $\left[p_{0}\right]$ and the failure indices presented in Table 2.

It is seen that the environment influences considerably on the strength of the studied composite plate.

\section{Conclusions}

The analysis presented in this paper, together with the computer code, can be used to determine the stress distribution and deformation of composite layered plates under combined mechanical and hygrothermal loads.

The results of this study show that the environment has significant influence on the mechanical behaviour of the graphite-epoxy composite plates. The stresses increase with elevated temperature and moisture concentration. Consequently, in the design of composite structures the influence of temperature and moisture on the strength of the structures needs to be thoroughly investigated. 
Tab. 2. The failure indices for the $\left(0^{\circ} / 90^{\circ} / 90^{\circ} / 0^{\circ}\right)$ plate under lateral and hygrothermal loads

\begin{tabular}{|c|c|c|c|c|}
\hline & $\begin{array}{c}\text { Case } 1 \\
(P)\end{array}$ & $\begin{array}{c}\text { Case } 2 \\
(P+M)\end{array}$ & $\begin{array}{c}\text { Case } 3 \\
(P+T)\end{array}$ & $\begin{array}{c}\text { Case } 4 \\
(P+T+M)\end{array}$ \\
\hline $\begin{array}{c}{\left[p_{0}\right]} \\
\Delta m_{S} ; \mathrm{DT} \\
\Delta T\end{array}$ & $\begin{array}{c}8.8 \mathrm{MPa} \\
0 \\
0\end{array}$ & $\begin{array}{c}8.19 \mathrm{MPa} \\
1 \% ; 1.2 \mathrm{~mm}^{2} \\
0\end{array}$ & $\begin{array}{c}7.79 \mathrm{MPa} \\
0 \\
50^{\circ} \mathrm{C}\end{array}$ & $\begin{array}{c}1.89 \mathrm{MPa} \\
1 \% ; 1.29 \mathrm{~mm}^{2} \\
50^{\circ} \mathrm{C}\end{array}$ \\
\hline Position & $0, b / 2, h / 4$ & $0, b / 2, h / 4$ & $0, b / 2,-h / 4$ & $a / 4, b / 4,-h / 4$ \\
\hline $\begin{array}{l}\sigma_{1}(\mathrm{MPa}) \\
\sigma_{2}(\mathrm{MPa}) \\
\sigma_{3}(\mathrm{MPa}) \\
\sigma_{23}(\mathrm{MPa}) \\
\sigma_{13}(\mathrm{MPa}) \\
\sigma_{12}(\mathrm{MPa})\end{array}$ & $\begin{array}{c}60.884941 \\
1.7350004 \\
-0.3882811 \\
-1.0313430 \\
99.294017 \\
-1.2552412\end{array}$ & $\begin{array}{c}68.040633 \\
2.2468071 \\
1.3362441 \\
-1.0340881 \\
97.674387 \\
-1.1714679\end{array}$ & $\begin{array}{c}73.799483 \\
3.2669914 \\
5.2838997 \\
-1.0402424 \\
93.862320 \\
-1.0647922\end{array}$ & $\begin{array}{c}27.457813 \\
12.282071 \\
44.601880 \\
-4.6597237 \\
0.2160413 \\
0.2808295\end{array}$ \\
\hline $\begin{array}{l}F_{1} \times \sigma_{1} \\
F_{2} \times \sigma_{2} \\
F_{3} \times \sigma_{3} \\
F_{11} \times \sigma_{1}^{2} \\
F_{22} \times \sigma_{2}^{2} \\
F_{33} \times \sigma_{3}^{2} \\
F_{44} \times \sigma_{23}^{2} \\
F_{55} \times \sigma_{13}^{2} \\
F_{66} \times \sigma_{12}^{2} \\
2 \times F_{23} \times \sigma_{2} \times \sigma_{3} \\
2 \times F_{13} \times \sigma_{1} \times \sigma_{3} \\
2 \times F_{12} \times \sigma_{1} \times \sigma_{2}\end{array}$ & $\begin{array}{c}-0.0081180 \\
0.0260240 \\
-0.0057212 \\
0.0019731 \\
0.0003010 \\
0.0000151 \\
0.0001064 \\
0.9857302 \\
0.0001576 \\
0.0000674 \\
0.0000788 \\
-0.0007715\end{array}$ & $\begin{array}{l}-0.0090721 \\
0.0337021 \\
0.0200437 \\
0.0024691 \\
0.0005048 \\
0.0001786 \\
0.0001069 \\
0.9540206 \\
0.0001372 \\
-0.0003002 \\
-0.0003031 \\
-0.0011064\end{array}$ & $\begin{array}{r}-0.0098399 \\
0.0490049 \\
0.0792285 \\
0.0029047 \\
0.0010273 \\
0.0027820 \\
0.0001082 \\
0.8802135 \\
0.0001134 \\
-0.0017262 \\
-0.0012998 \\
-0.0017608\end{array}$ & $\begin{array}{c}-0.0036610 \\
0.1842311 \\
0.6690262 \\
0.0004021 \\
0.0150829 \\
0.1989328 \\
0.0021713 \\
0.0000047 \\
0.0000079 \\
-0.0546803 \\
-0.0040822 \\
-0.0024628\end{array}$ \\
\hline Failure Index & 1 & 1 & 1 & 1 \\
\hline
\end{tabular}

\section{REFERENCES}

1. Noor A. K., Burton W. S. and Bert C. W. Computational model for sandwich panels and shells. Applied mechanics reviews, Vol.49, N.3̈, March 1996, pp. $55-199$.

2. Noor A. K., Starnes J. H. and Peters J. M. Analysis of curved sandwich panels subjected to combined temperature gradient and mechanical loads. Proceedings 
of the AIAA/ASME/ASCE/AHS/AC structure, structural dynamics and materials conference 1997, Vol. 4, AIAA, New York, pp. 2446-2470.

3. Noor A. K and Peters J. M. Analysis of curved sandwich panels with cutouts subjected to combined temperature gradient and mechanical loads in Proceedings of the ASME Aerospace division, structures and materials commitee, Editor, G.J. Simites, ASME-AD- Vol.55, New York 1997.

4. Tran Ich Thinh, Ngo Nhu Khoa. Modelling the mechanical and hygrothermal behaviour of composite laminates using a high-order displacement formulation, Proceedings of the International Coloquium on Mechannics of Solids, Fluids, Structures and interaction. Nhatrang Vietnam 14-18/8/2000.

5. Tran Ich Thinh, Ngo Nhu Khoa. Hygroscopically induced residual stresses in laminated composites. Vietnam Journal of Mechanics, Vol.23, No. 3, 2001.

6. Ngo Nhu Khoa, Tran Ich Thinh. On a bending problem of thick laminated Composite Plates. Vietnam Journal of Mechanics, Vol. 24, No. 1, 2002.

7. Tsai S. W. and Wu E. M. A General Theory of Strength for Anisotropic Materials. J. Composite Materials, Vol. 5, 1971, PP. 58-80.

Received August 12, 2002

ƯNG XƯ CO - NHIỆT Å̉M CỦA TẤM COMPOSITE LỚP

Bài báo nghiên cứu ứng xư cơ - nhiệt ẩm của tấm composite lớp chữ nhật, tựa bản lề trên 4 cạnh. Quá trình phân tích sử dụng phương pháp phần tử hữu hạn và dựa vào lý thuyết chuyển vị bậc 3 đầy đủ. Các ví dụ số được minh họa cho tấm bằng composite lớp đối xứng, "đúng trục": $\left(0^{\circ} / 90^{\circ} / 90^{\circ} / 0^{\circ}\right)$ và "lệch trục": $\left(45^{\circ} /-45^{\circ} /-45^{\circ} / 45^{\circ}\right)$. 